A0024 Effect of Combination of Ketamine and Propofol (Ketofol) on Cerebral Oxygenation Using $\mathrm{SjVO}_{2}$ as Parameter in Neurosurgical Patients: A Randomized Double-Blind Control Trial

Vishwanatha, ${ }^{1}$ Nidhi Panda, ${ }^{1}$ Hemant Bhagat, ${ }^{1}$ Rajesh Chhabra, ${ }^{2}$ Ankur Luthra ${ }^{1}$

${ }^{1}$ Department of Anaesthesia and Intensive Care, Postgraduate Institute of Medical Education and Research, Chandigarh, India ${ }^{2}$ Department of Neurosurgery, Postgraduate Institute of Medical Education and Research, Chandigarh, India

Background: The effects of ketofol on several parameters such as hemodynamics and requirement of opioids/ propofol have been published. But data on effects of ketofol on cerebral oxygenation are inadequate. This study aims to compare the effects of ketofol (ketamine:propofol = 1:5) with propofol on cerebral oxygenation (jugular venous oxygen saturation $\left.\left[\mathrm{SjVO}_{2}\right]\right)$, in patients undergoing surgical clipping of intracranial aneurysm.

Materials and Methods: Forty WFNS I and II patients were randomized into ketofol $(n=20)$ and propofol $(n=20)$ groups. Post-induction $\mathrm{SjVO}_{2}$ catheter was inserted, and anesthesia was maintained with propofol/fentanyl in propofol group and ketofol/fentanyl in the ketofol group. $\mathrm{SjVO}_{2}$ was obtained at baseline, 1 hour and 2 hours intraoperatively and at 6 hours after the surgery. Also, intraoperative brain relaxation, fentanyl requirement, and hemodynamics were noted.

Results: Higher $\mathrm{SjVO}_{2}$ values were observed in ketofol group compared with propofol at 1 and 2 hours after starting the drug and at 6 hours after surgery $(p<0.05)$. In propofol group, a significant fall in $\mathrm{SjVO}_{2}$ was recorded at 2 hours (during temporary clipping) after the starting of drug as compared with the baseline $(p=0.001)$. All recordings of $\mathrm{SjVO}_{2}$ in both the groups were within normal limits. Requirement of fentanyl in ketofol group was less as compared with propofol group $(p=0.022)$. More than $20 \%$ fall in mean arterial pressure (MAP) compared with baseline MAP was noted in $75 \%$ of patients in propofol group and 15\% of patients in ketofol group $(p=0.002)$. In propofol group, 55\% patients required rescue drug phenylephrine to treat hypotension, whereas only $15 \%$ patients required it in ketofol group $(p=0.02)$. Brain relaxation score was comparable in both the study groups ( $p=0.887$ ).

Conclusions: Maintenance of anesthesia with ketofol provides better cerebral oxygenation and hemodynamic stability compared with propofol in patients undergoing clipping of cerebral aneurysm after SAH.

\section{A0025 An Unusual Case Presentation as Nonconvulsive Status Epilepticus: Autoimmune Encephalitis \\ Deepak Solanki, ${ }^{1}$ Saurabh Anand ${ }^{1}$ \\ ${ }^{1}$ Department of Neuroanaesthesiology and Neurocritical Care, Artemis hospital, Gurugram, Haryana, India}

Background: Nonconvulsive status epilepticus (NCSE) accounts for 25\% of all cases of status epilepticus. Refractory status epilepticus (RSE) is defined as continued clinical/electrographic seizures after adequate dosing of initial benzodiazepine, followed by second-line antiepileptic drug and is associated with worse prognosis than nonrefractory status epilepticus. When RSE occurs in an individual without any history of epilepsy and no immediate underlying etiology is found, it is referred to as new-onset refractory status epilepticus (NORSE). This clinical scenario may be notoriously difficult to treat and does not respond to initial medications. In cases of NORSE where an etiology is found, antibody-mediated disorders are the most common cause. Autoimmune encephalitis refers to a diverse group of neuropsychiatric disorders. It can have a variable pattern of clinical presentation with an array of symptoms many of which make diagnosis difficult due to similarities in clinical, imaging, and laboratory findings with respect to other forms of autoimmune or infectious encephalitis.

Case Description: Our case report highlights how a patient with acute-onset history, showing generalized periodic epileptiform discharges on electroencephalogram (EEG) but with no prior history of seizures was eventually diagnosed as autoimmune encephalitis based on clinical findings, cerebrospinal fluid (CSF) reports, and EEG analysis.

Conclusions: As a presentation of autoimmune encephalitis, nonconvulsive status epilepticus requires high level of suspicion. Early EEGs with prompt and aggressive treatment can improve patient outcomes drastically.

\section{A0026 Diagnosis of Nonalcoholic Wernicke Encephalopathy in an Intensive Care Unit Patient: A Diagnostic Dilemma}

Sonika Bishnoi, ${ }^{1}$ Swati, ${ }^{1}$ Satinder Gombar,' Vanita Ahuja, ${ }^{1}$ Vinod Bishno ${ }^{2}$

${ }^{1}$ Department of Anesthesia and Intensive Care, Government Medical College and Hospital, Postgraduate Institute of Medical Education and Research, Chandigarh, India.

${ }^{2}$ Department of Anesthesia and Intensive Care, Postgraduate Institute of Medical Education and Research, Chandigarh, India.

Background: Wernicke encephalopathy (WE) is a known complication of thiamine deficiency, normally seen in patients having excessive alcohol intake. It is characterized by a triad of symptoms of confusion, ophthalmoplegia, and ataxia. Symptomatic thiamine deficiency in nonalcoholics is less known (0.1\%) and is often underdiagnosed. Overall, if WE is diagnosed at an early stage and treated, complications can be reversed. We present the case of a patient operated for perforation peritonitis who was subsequently diagnosed and managed for nonalcoholic WE.

Case Description: A 22-year-old male patient admitted to surgical emergency with complain of pain abdomen and vomiting since 2 days survived after an event of cardiac arrest. Patient was operated for perforation peritonitis and shifted to intensive care unit (ICU). On 35th day of ICU stay, he became disoriented and developed nystagmus. A provisional diagnosis of neurological sequelae of hypoxic ischemic brain injury was made, and MRI was planned. MRI showed features suggestive of WE. Intravenous thiamine was started. The patient improved, became conscious, responsive, and nystagmus disappeared. Gradually, the patient was weaned to room air and discharged home.

Conclusions: Wernicke encephalopathy should be taken into consideration in surgical patients admitted in 
ICU. Nonalcoholic WE should be considered as a possible diagnosis when the classic triad is absent. Prognosis of the patient mostly depends on early recognition of the syndrome and early initiation of thiamine treatment. It may be fatal if it progresses to Korsakoff syndrome. Thus, higher suspicion and recognition of WE in early stage can increase chances of full reversal of this condition.

\section{A0027 Patient with Hurler's Syndrome for Emergency Ventriculoperitoneal Shunt: What an Anesthesiologist Must Know \\ Teena Bansal, ${ }^{1}$ Shashi Kiran, ${ }^{1}$ Savita Saini, ${ }^{1}$ Tarun Yadav ${ }^{1}$ ${ }^{1}$ Department of Anaesthesiology and Critical Care, Pt. B. D. Sharma Post Graduate Institute of Medical Sciences, Rohtak, Haryana, India}

Background: Patients with Hurler's syndrome pose a great challenge to anesthesiologists. The most common problem faced by anesthesiologist is difficult venous access and establishment and maintenance of difficult airway.

Case Description: A 22-year-old female patient, a known case of Hurler's syndrome, presented with complaint of headache. She was scheduled for insertion of VP shunt for acute hydrocephalus. History was significant for obstructive sleep apnea, and she used CPAP during sleep for the same. The patient was cooperative but slow in response to commands. General physical examination revealed short stature, dysmorphic face, macrocephaly, frontal bossing, short neck, depressed nasal bridge, dorsolumbar kyphosis, flexion deformity of elbows and wrists, and stubby fingers. Airway evaluation revealed large tongue and Mallampati grade IV. Echocardiography revealed mitral valve prolapse with trivial mitral regurgitation. Intravenous access was obtained with difficulty. Difficult mask ventilation was anticipated, in addition to difficult laryngoscopy and intubation. Awake fiberoptic intubation was performed successfully. Anesthesia was induced, and at the end of surgery, trachea was extubated awake.

Conclusions: The most important step in management of airway is to avoid a situation of "can't ventilate, can't intubate." The basic aim is preservation of spontaneous ventilation and is of utmost importance. Recovery after general anesthesia is often slow in these patients and may be accompanied by breath-holding, apnea, and bronchospasm. The patient should be awake with adequate airway reflexes for extubation.

\section{A0028 Effect of Comorbidities on the Outcome of Patients with Aneurysmal Subarachnoid Hemorrhage: A Prospective Observational Study \\ Sunaakshi Puri, ${ }^{1}$ Hemant Bhagat, ${ }^{1}$ Kiran Jangra \\ ${ }^{1}$ Department of Anaesthesia and Intensive Care, Postgraduate Institute of Medical Education and Research, Chandigarh, India}

Introduction: Subarachnoid hemorrhage (SAH) carries a high mortality of 30 to $40 \%$. Amongst survivors, 40 to $50 \%$ suffer disability and cognitive decline. Comorbidities can have a contribution in the disease process. We hypothesize that the presence of comorbidities in a patient with aneurysmal SAH can influence the course of disease and thereby the neurological outcome.

Materials and Methods: This prospective observational study was commenced after ethical committee clearance and written informed consent. We enrolled 140 (94 females and 46 males) consecutive patients with aneurysmal SAH, scheduled for clipping/coiling from July 2016 to October 2017. Patients' comorbidities were recorded and outcome followed in postoperative period using MRS at discharge and mRS and GOS at 1 month after discharge. Analysis was done using chi-square test.

Results: In patients undergoing clipping, HTN and smoking are associated with worse outcome (0.02 and 0.01 , respectively). HTN is associated with increased incidence of vasospasm and infarct while smoking and CAD are associated with increased incidence of rebleed and hydrocephalus, respectively. In patients undergoing coiling, HTN, smoking, and CAD are associated with worse outcome $(0.02,0.04$, and 0.02 , respectively). Smoking, alcohol intake, and CAD are associated with increased incidence of rebleed while CAD is associated with increased incidence of hydrocephalus. DM, thyroid dysfunction, TB, and joint disease are not associated with worse outcome or increased incidence of complications.

Conclusions: Various comorbidities including HTN, CAD, smoking, and alcohol intake were associated with increased risk of perioperative complications in patients after $\mathrm{SAH}$. Other comorbidities such as DM, thyroid dysfunction, $\mathrm{TB}$, and joint disease neither affect outcome nor increase risk of complications.

A0029 Measurement of Neutrophil GelatinaseAssociated Lipocalin for Acute Kidney Injury following Neuroradiological Procedure(s) in Patients with Aneurysmal Subarachnoid Hemorrhage: A Preliminary Study

Ashutosh Kaushal, ${ }^{1}$ Ashish Bindra, ${ }^{1}$ Rajendra S. Chouhan ${ }^{1}$ ${ }^{1}$ Department of Neuroanaesthesiology and Critical Care, All India Institute of Medical Sciences, New Delhi, India

Background: Patients with aneurysmal SAH are susceptible to acute kidney injury (AKI) due to preexisting hypovolemia, renal hemodynamic changes, and neuroendocrine response. NGAL represents a promising biomarker for early prediction, monitoring treatment response, and prognostication of AKI in various clinical settings. The purpose of this study was to find incidence of AKI and to see changes in plasma NGAL at different time periods in aneurysmal SAH patients undergoing neuroradiological intervention.

Materials and Methods: All consenting aneurysmal SAH patients (18-65 years, modified Hunt \& Hess grade 1-4) posted for neuroradiological procedures during the study time were included. Patients with history of chronic renal disease, recent contrast exposure, and renal insufficiency due to any other reasons were excluded. Blood sample for serum creatinine and serum NGAL were obtained preprocedure and then at 1, 6, 24, and 48 hours after contrast administration. AKI was defined by KDIGO clinical practice guidelines. 